than 80 percent of the predicted value). Accordingly, the results of the SUPER study cannot be extrapolated for patients with pulmonary hypertension related to lung diseases.

Drs. Hoeper and Welte are correct in their suggestion that there is a linear trend in the response among the doses in the hemodynamic measures and in the percentages of patients who improved by at least one WHO functional class. The absence of a dose-response effect on the primary end point (exercise capacity) or on tolerability with the three sildenafil doses $(20 \mathrm{mg}, 40 \mathrm{mg}$, and $80 \mathrm{mg}$ three times daily) may be related to complete inhibition of phosphodiesterase type 5 with the lowest dose. However, in the extension study, all patients were treated with $80 \mathrm{mg}$ three times daily (if tolerated), and we do not have prospective information on the long-term efficacy of the approved dose of $20 \mathrm{mg}$ three times daily. Based on these data, $20 \mathrm{mg}$ three times daily appears to be a reasonable initial dose; an increase to $40 \mathrm{mg}$ three times daily, $80 \mathrm{mg}$ three times daily, or both may be considered in order to achieve or maintain favorable effects. We recognize that limited data are currently available regarding the optimal dose of sildenafil for long-term treatment and that the use of higher doses may be hampered by the restricted approval.

Nazzareno Galiè, M.D.

University of Bologna

40138 Bologna, Italy

nazzareno.galie@unibo.it

Lewis J. Rubin, M.D.

University of California at San Diego

La Jolla, CA 92103-8372

Gérald Simonneau, M.D.

Hopital Antoine Béclère

92140 Clamart, France

1. Barst RJ, Rubin LJ, Long WA, et al. A comparison of continuous intravenous epoprostenol (prostacyclin) with conventional therapy for primary pulmonary hypertension. N Engl J Med 1996; 334:296-302.

2. Simonneau G, Galie N, Rubin LJ, et al. Clinical classification of pulmonary hypertension. J Am Coll Cardiol 2004;43:Suppl $12: 5 S-12 S$.

\title{
Congenital Glutamine Deficiency with Glutamine Synthetase Mutations
}

TO THE EDITOR: Häberle et al. (Nov. 3 issue) ${ }^{1}$ state that "the concentration of glutamate . . . was not elevated in either of the two patients, but this lack of elevation might be due to the various other pathways of glutamate catabolism." We suggest that low-to-deficient glutamate concentrations in cerebrospinal fluid indicate a chronic depletion of neuronal glutamate stores, since astrocytic glutamine synthase is crucial for their replenishment. After the bioenergetic coupling of and metabolite trafficking between astrocytes and neurons occur, ${ }^{2}$ a complete deficiency of this enzyme should result in a severe depletion of neuronal glutamate stores in the long run and, subsequently, a drain of intermediates of the tricarboxylic acid cycle due to increased new synthesis of glutamate and insufficient anaplerosis through pyruvate carboxylation in neurons. ${ }^{3}$ As a consequence, concentrations of glutamate in the cerebrospinal fluid may decrease, as seen in both patients mentioned above. Neurotransmission of glutamate is important for the developing brain, for modulating neuronal migration, for growth spurts of the brain, and for apoptosis. ${ }^{4}$ Dysfunction of these mechanisms probably induces brain damage and malformations, ${ }^{5,6}$ as displayed in both patients.

Stefan Kölker, M.D.

Georg F. Hoffmann, M.D.

Jürgen G. Okun, Ph.D.

University Children's Hospital

D-69120 Heidelberg, Germany

stefan_koelker@med.uni-heidelberg.de

1. Häberle J, Görg B, Rutsch F, et al. Congenital glutamine deficiency with glutamine synthetase mutations. N Engl J Med 2005; 353:1926-33.

2. Magistretti PJ, Pellerin L, Rothman DL, Shulman RG. Energy on demand. Science 1999;283:496-7.

3. Waagepetersen HS, Qu H, Schousboe A, Sonnewald U. Elucidation of quantitative significance of pyruvate carboxylation in cultured cerebellar neurons and astrocytes. J Neurosci Res 2001;66:763-70.

4. Komuro H, Rakic P. Modulation of neuronal migration by NMDA receptors. Science 1993;260:95-7.

5. Ikonomidou C, Bosch F, Miksa M, et al. Blockade of NMDA receptors and apoptotic neurodegeneration in the developing brain. Science 1999;283:70-4.

6. Ikonomidou C, Bittigau P, Ishimaru MJ, et al. Ethanol-induced apoptotic neurodegeneration and fetal alcohol syndrome. Science 2000;287:1056-60. 
TO THE EDITOR: We read with interest the report by Häberle et al., which indicates that systemic glutamine deficiency due to a glutamine synthetase gene mutation results in brain malformations and seizures. An additional explanation might be related to chronic hyperammonemia resulting from a deficiency of glutamine synthetase. However, levels of ammonia were not reported. Glutamine synthetase not only produces glutamine but also detoxifies ammonia. ${ }^{1}$ In the brain, glutamine synthetase fuels glutamate uptake by astrocytes; therefore, inhibition of glutamine synthetase results in increased extracellular glutamate levels and excitotoxic effects, leading to seizures. ${ }^{2}$ Hyperammonemia in the setting of liver failure leads to neurologic impairment. Also, infants with enzyme abnormalities of the urea cycle, such as a deficiency of ornithine carbamoyltransferase, who survive episodes of hyperammonemia have a high incidence of mental retardation and cerebral palsy. ${ }^{3}$ In patients with lung transplants, hyperammonemia resulting from hepatic glutamine synthetase deficiency is associated with high mortality. ${ }^{4}$ The observation of increased glutamine synthetase protein levels in the presence of reduced activity supports the view that hyperammonemia may set up a self-perpetuating cycle whereby ammonia can reduce glutamine synthetase activity. ${ }^{5}$ Therefore, it is possible that the inhibition of glutamine synthetase in the brain may lead to increased levels of ammonia and extracellular glutamate in the brain, partly explaining the clinical observations.

Christopher Rose, Ph.D.

Fundacion Valenciana de Investigaciones Biomedicas 46013 Valencia, Spain

Rajiv Jalan, M.D., Ph.D.

University College London Institute of Hepatology

London WClE 6HX, United Kingdom

r.jalan@ucl.ac.uk

1. Martinez-Hernandez A, Bell KP, Norenberg MD. Glutamine synthetase: glial localization in brain. Science 1977;195:1356-8.

2. Shaked I, Ben-Dror I, Vardimon L. Glutamine synthetase enhances the clearance of extracellular glutamate by the neural retina. J Neurochem 2002;83:574-80.

3. Maestri NE, Brusilow SW, Clissold DB, Bassett SS. Long term treatment of girls with ornithine transcarbamylase deficiency. N Engl J Med 1996;335:855-9.

4. Lichtenstein GR, Yang YX, Nunes FA, et al. Fatal hyperammonemia after orthotopic lung transplantation. Ann Intern Med 2000;132:283-7.

5. Schliess F, Gorg B, Fisher R, et al. Ammonia induces MK801-sensitive nitration and phosphorylation of protein tyrosine residues in rat astrocytes. FASEB J 2002;16:739-41.
THE AUTHORS REPLY: In their letter, Dr. Kölker and colleagues comment on the low glutamate concentrations in cerebrospinal fluid in patients with glutamine synthetase deficiency. The authors suggest that chronic depletion of neuronal glutamate stores is a result of the defective glutamine-glutamate cycle. This is an interesting speculation and refers to the need for the investigation of neurotransmitters in cerebrospinal fluid during the follow-up of patients with glutamine synthetase deficiency, which has not been done in the case of the patients described in our article. However, the low glutamate concentrations in cerebrospinal fluid were established with the use of chromatography, which is not sufficiently sensitive to define glutamate concentrations accurately in the very low range. Therefore, it is not possible to include a deficiency of glutamate in cerebrospinal fluid as one of the features of glutamine synthetase deficiency.

The comments of Drs. Rose and Jalan refer to the levels of ammonia in the patients described. The authors speculate that hyperammonemia was present as a result of the absence of detoxification with the glutamine synthetase reaction. The plasma ammonia level in Patient 1 was $77 \mu \mathrm{mol}$ per liter. In Patient 2, the ammonia level was $140 \mu \mathrm{mol}$ per liter, a finding that, in fact, led to further metabolic investigations, including the amino acid profile. During the course of the disease, however, plasma ammonia levels were normal. Thus, hyperammonemia cannot be regarded as a sign of glutamine synthetase deficiency. It would also not explain the severe malformation of the brain and extracerebral manifestations - for example, cutaneous manifestations. The comment regarding the increased levels of extracellular glutamate leading to excitotoxic effects is difficult to reconcile with the findings of low glutamate levels in the cerebrospinal fluid of these patients.

Johannes Häberle, M.D.

Universitätsklinikum Münster 48129 Münster, Germany

Freimut Schliess, Ph.D.

Dieter Häussinger, M.D.

Heinrich-Heine-Universität Düsseldorf 40225 Düsseldorf, Germany 